\title{
Application of the Projective Standard Deviation to STEM Imaging and Analysis
}

\author{
Everett D. Grimley ${ }^{1}$, Xiahan Sang ${ }^{1}$ and James M. LeBeau ${ }^{1}$ \\ ${ }^{1 .}$ Department of Materials Science and Engineering, North Carolina State University, Raleigh, NC
}

The radon transformation has demonstrated tremendous utility for the processing of tomographic reconstructions, though it remains largely unapplied to problems of imaging in scanning transmission electron microscopy (STEM). A complete radon transformation projects image intensities onto lines oriented at different angles relative to the image. When orientated normal to a lattice vector, the projection becomes dominated by the intensity fluctuations determined by the interplanar spacing, as seen in the red trace in Figure 1. Projections away from these conditions result in flatter intensities as shown in the black trace in Figure 1. The standard deviation of these projected intensities thus scales with the degree to which an angle corresponds to a lattice vector. We have recently shown that the standard deviation of the normalized radon transformation as a function of angle, herein called the projective standard deviation (PSD), serves as a critical tool to enable the elimination of drift related distortions using the revolving-STEM (RevSTEM) method [1]. Furthermore, its utility extends far beyond the RevSTEM method.

In this talk, we will report the use of PSD as a tool to analyze STEM images. We will emphasize the analytical power of PSD by discussing a technique for accurate and simple measurement of electron probe size and shape. We will also highlight the general sensitivity of PSD to atomic structure, which affords many useful applications to STEM imaging and analysis. For example, Figure 2 (a) shows a PSD calculated from a simulated $\mathrm{Si}<100>$ HAADF-STEM image; note the sharp peaks and fine lobe features. We will show that when simulated HAADF-STEM images are convolved with different twodimensional Gaussian distributions, radically different PSDs result. We will demonstrate that the PSD exhibits acute sensitivity to the electron probe shape and to the combined contributions of the finite effective source size and the coherent aberrations. Comparison between the PSDs of experimental images (probe-corrected FEI Titan G2 60-300 keV with convergence semi-angle of 15 mrad) and PSDs of simulated HAADF-STEM images allows the characterization of the probe shape. As an example, the PSD of an experimental Si $<100>$ HAADF-STEM image (background subtracted) shown in Figure 2 (b) was compared with the PSDs of simulated images convolved with Gaussian functions of different parameters. After fitting, the corresponding simulated image, Figure 2 (c), is nearly identical to the experimental data, shown in Figure 2 (d).

The sensitivity of PSD to structure renders it an invaluable tool for analyzing many useful crystallographic properties of a specimen. Beyond probe size estimation, we will also demonstrate that the technique can be used to index atom column locations, which allows many important analyses to become simple array operations, such as distance mapping and calculating the ratios of atom column intensities [2].

References:

[1] X Sang and JM LeBeau, Ultramicroscopy 138 (2014), p. 28 
[2] The authors acknowledge the use of the Analytical Instrumentation Facility (AIF) at North Carolina State University, which is supported by the State of North Carolina and the National Science Foundation. The authors also acknowledge use of the High Performance Computing (HPC) services at North Carolina State University.

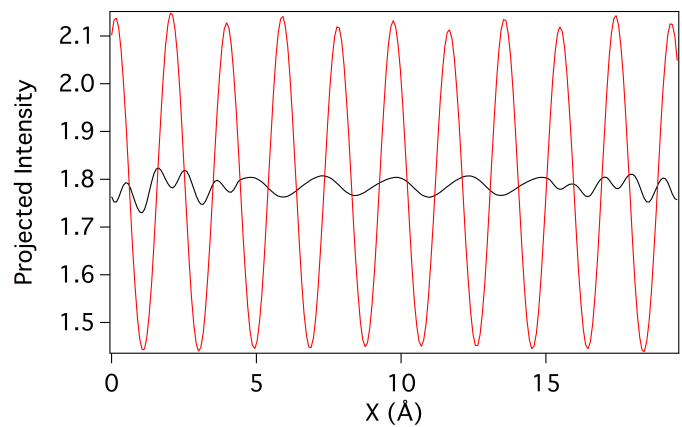

Figure 1. Projected image intensities of a simulated HAADF-STEM image of $\mathrm{Si}<100>$ at angles normal to a lattice vector (red) and off-normal to a lattice vector (black).

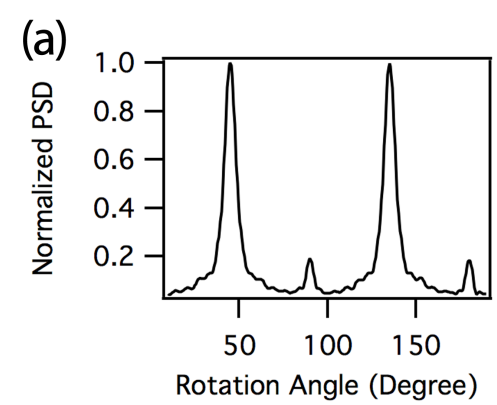

(c)

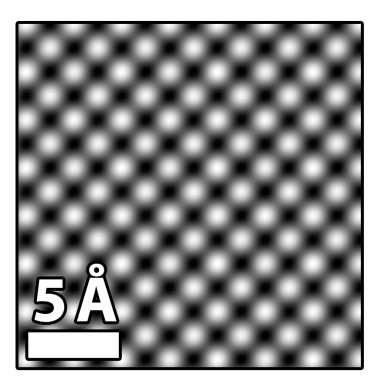

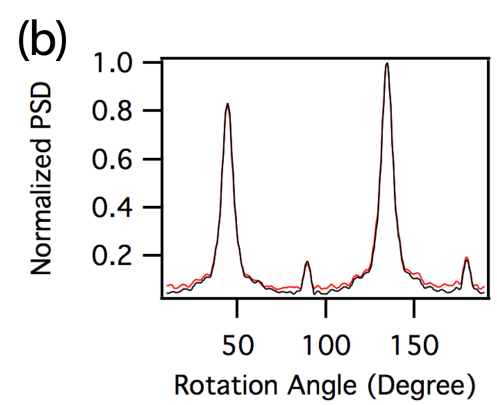

(d)

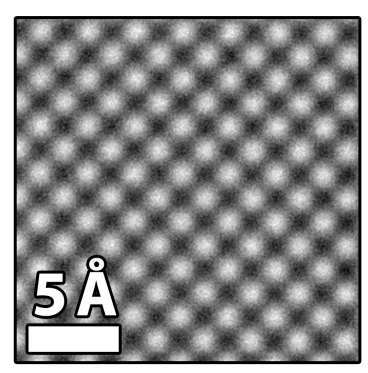

Figure 2. (a) PSD of a simulated $\mathrm{Si}<100>$ HAADF-STEM image convolved with a Gaussian function (FWHM $1.0 \AA$ by $1.0 \AA$ and no rotational component) over the angle range $10^{\circ}$ to $190^{\circ}$. (b) PSD of an experimental Si $<100>$ RevSTEM HAADF-STEM image (red trace) and PSD of a simulated $\mathrm{Si}<100>$ HAADF-STEM image (black trace) convolved with a Gaussian function (FWHM $1.02 \AA$ by $0.92 \AA$ and rotational component $\sim \pi / 4$ radians). (c) Simulated HAADF-STEM Si $<100>$ image convolved with a Gaussian function using parameters from the fit in (b). (d) Aberration corrected HAADF-STEM Si $<100>$ image (background subtracted using a Fourier-space approach) used for the experimental PSD in (b). 